\title{
Concentración de medios o concentración de temas en el periodismo digital peruano. Un acercamiento cuantitativo al problema
}

Concentration of media or concentration of topics in Peruvian digital journalism. A quantitative approach to the problem

MIGUEL SÁNCHEZ FLORES

Periodista, escritor y docente universitario. Licenciado en periodismo y magíster en Historia del Arte y Curaduría, ambos grados otorgados por la Pontificia Universidad Católica del Perú (PUCP). Estudios de Literatura en la Universidad Nacional Mayor de San Marcos. Actualmente, doctorando de Literatura Hispanoamericana en la PUCP. Ha desarrollado investigaciones sobre educación, periodismo digital y arte peruano contemporáneo, y forma parte del grupo de investigación Comunicación y Subjetividades, que busca observar y problematizar las diferentes subjetividades en el arte, las culturas digitales, el activismo ciudadano, las industrias culturales y la comunicación en los márgenes en contextos de globalización, neoliberalismo y posconflicto. Ha publicado el libro de cuentos Ciudades vencidas (Animal de Invierno, 2016) y la novela Secta Pancho Fierro (Planeta, 2017), con la cual obtuvo el 8 vo. Premio de Novela Breve de la Cámara Peruana del Libro. 



\title{
Concentración de medios o concentración de temas en el periodismo digital peruano. Un acercamiento cuantitativo al problema Concentration of media or concentration of topics in Peruvian digital journalism. A quantitative approach to the problem
}

\author{
Miguel Sánchez Flores \\ PONTIFICIA UNIVERSIDAD CATÓLICA DEL PERÚ \\ sanchez.miguel@pucp.edu.pe
}

\section{PALABRAS CLAVE / KEYWORDS}

Periodismo / Prensa peruana / Periodismo digital / Concentración de medios / Contenidos periodísticos

Journalism / Peruvian press / Digital journalism / Media conglomerates/ Journalistic content

\section{SUMILLA}

Este artículo presenta algunos de los principales hallazgos del proyecto de investigación “¿Concentración de medios o concentración de temas en el periodismo peruano? Un análisis cuantitativo de los contenidos de 3 sitios web de noticias del Perú (El Comercio, La República y Radio Programas del Perú)", cuyo objetivo fue analizar el trabajo de producción de contenidos propios dentro de tres de las redacciones digitales más importantes del país y, con ello, comprobar la variedad informativa que existe dentro del mercado del periodismo digital peruano. Asimismo, este texto presenta la metodología utilizada y analiza los resultados de la investigación, poniendo especial énfasis en dos de las cuatro categorías utilizadas: "secciones" y "fuentes de procedencia". El estudio además revela si en el Perú no solo asistimos a una concentración de medios de comunicación en el sector impreso, donde el Grupo El Comercio maneja cerca del $80 \%$ del mercado de circulación nacional, sino también ante una concentración de temas en el que los principales medios digitales "compiten" por la audiencia utilizando los mismos contenidos y las mismas estrategias para ello.

\section{ABSTRACT}

This article presents some of the main findings of the research project "Concentration of media or concentration of topics in Peruvian journalism? An analysis of the contents of the 3 news websites in Peru (El Comercio, La República and Radio Programas del Perú), whose objective was to analyze the work of producing their own content in three of the most important digital newsrooms of the country 
and, with it, verify the variety of information that exists inside the Peruvian digital journalism market. Likewise, the text presents the proposed methodology and analyzes the results of the research, making special emphasis on two of the four study categories: "sections" and "sources of origin". The investigation reveals if in Peru, we not only attend a media concentration in the printed sector, where the El Comercio Group handles close to $80 \%$ of the national circulation market, but also exists a concentration of topics in which the main digital media "compete" for the audience using the same content and the same strategies for it.

\section{Concentración de temas}

Mucho se habla sobre la crisis de los contenidos del periodismo digital peruano, pero poco es lo que se ha estudiado sobre el tema. En ese sentido, la presente investigación, cuyos algunos de sus alcances detallamos en este texto, se presenta aún como un acercamiento metodológico que busca entregar información cuantitativa que explique dicha problemática y que también sirva como una línea de base para futuras investigaciones sobre el tema.

La investigación ${ }^{1}$, que se desarrolló entre septiembre y diciembre del año 2015, propuso corroborar la poca variedad de los temas de la prensa digital peruana, específicamente de los portales peruanos El Comercio (elcomercio.pe), La República (larepublica.pe) y Radio Programas del Perú (rpp.pe), tres de los principales medios de comunicación del país.

El estudio partió de la constatación de que muchos de estos contenidos, de fácil publicación y de escasa elaboración, priorizaban, entre otras, las notas de agencias de noticias, los videos de programas televisivos locales, las notas de web extranjeras, los listados, los videos virales de YouTube o los memes generados en redes sociales. Se buscaba corroborar que eran estos contenidos -similares y de fácil producción- los que conformaban el corpus de la prensa digital peruana en dicho período, indistintamente del medio.

El objetivo principal de la investigación entonces fue: analizar el trabajo de producción de contenidos propios dentro de los sitios El Comercio, La República y Radio Programas del Perú (RPP) y, con ello, comprobar la variedad informativa que existe dentro del mercado del periodismo digital peruano. La propuesta era trascender la discusión de la concentración de medios ${ }^{2}$, centrándola también en el riesgo de concentrar temas y estrategias, incluso

\footnotetext{
1 La investigación recibió el financiamiento de la Dirección de Gestión de la Investigación del Vicerrectorado de Investigación de la Pontificia Universidad Católica del Perú, gracias a que fue uno de los proyectos ganadores del Concurso Anual de Proyectos 2015. Contó con la asistencia de las alumnas de la Facultad de Ciencias y Artes de la Comunicación, Kuyayki Zapata y Guadalupe Díaz.

2 Para Jorge Acevedo (2013), la compra del 54\% de acciones de Epensa por el Grupo El Comercio profundizó "los niveles de concentración mediática en el Perú" y configuró "una barrera que limita el ejercicio del derecho a la comunicación y la diversidad política y cultural en el espacio público".
} 
en casas periodísticas distintas, como es el caso de los tres medios seleccionados para la investigación.

A su vez, los objetivos específicos que se propusieron en esta investigación fueron los siguientes:

- Conocer cuáles son las secciones, géneros, formatos y fuentes más comunes en las noticias que se publican en los sitios digitales de El Comercio, La República y RPP.

- Comparar y clasificar los contenidos publicados diariamente por $\mathrm{El} \mathrm{Co}$ mercio, La República y RPP.

- Conocer cuál es el porcentaje de contenido propio que se genera en las redacciones estudiadas.

- Conocer las herramientas 2.0, su funcionamiento y alcance, así como sus limitaciones, en función al papel de los medios tradicionales.

\section{Pertinencia: estudios cuantitativos y prensa digital}

Si bien el campo de estudios en relación a la prensa digital en el Perú aún está poco desarrollado, en otros países ya existe una detallada tradición de investigación y de bibliografía, sobre todo vinculada a tópicos relacionados a la propia metodología, la evolución de la prensa digital y sus contenidos, y la importancia de esta en la fijación de agendas, así como en temas más específicos como la integración de redacciones, las posibilidades de lo multimedia y las estrategias de posicionamiento web.

De larga tradición, el análisis de contenidos es una de las técnicas más utilizadas en los estudios de la comunicación social. Tal como lo señala José Ignacio Ruiz (2012), si la observación es el más espontáneo y antiguo modo de recoger información, y la entrevista el más popularizado para los investigadores actuales, el análisis de contenidos es el más amplio, universalizado y rico de los métodos. Sin embargo, sobre este existen muchas definiciones, siendo la de Kerlinger (1979) una de las más clásicas. Este autor sostiene que al análisis de contenidos es un método que estudia y analiza la comunicación de una manera sistemática, objetiva y cuantitativa, con el propósito de encontrar variables de medición. Una investigación de este tipo fue desarrollada por Javier Odriozola y Guillermo López, de la Universitat Pompeu Fabra de España, quienes publicaron los siguientes estudios: "Content Analysis applied to Digital Media: A Comparison of News in 'The Guardian', 'Clarín', and 'Asahi Simbun'” (2011) y "Análisis de contenido de los cibermedios generalistas españoles. Características y adscripción temática de las noticias principales de portada” (2010).

En dichas investigaciones los autores hacen un análisis de contenidos tomando 
en cuenta algunas variables específicas como: i) distribución espacial, ii) presencia audiovisual, iii) interactividad, iv) secciones, v) autores, vi) temas principales, $\mathrm{y}$ vii) números de fuentes usadas. Para ello, utilizan formulaciones matemáticas -el índice Kappa de Cohen y el método de Holsti- para organizar y tabular los resultados de una observación de meses de diversos sitios web del mundo, centrando su atención en los homes. Los resultados encontrados son significativos y señalan, por ejemplo, que en el caso de los medios españoles, las portadas web -en su mayoría- son divididas en dos columnas, siendo las secciones "política" y "deportes" las más ofrecidas en los espacios de mayor visibilidad.

Los estudios mencionados son relevantes en la medida en que el método utilizado en ellos se acerca mucho al que siguió la investigación aquí expuesta; sin embargo, también es importante destacar algunas de sus especificidades. A diferencia de las investigaciones mencionadas, lo que buscaba nuestro estudio era medir el total de publicaciones ${ }^{3}$, sean estas, o no, de portada. Al no poder saber qué hace la audiencia al navegar por las páginas analizadas (como sí lo saben los editores de los sitios web a través de servicios de pago, tipo Google Analytics), lo que nos interesaba era indagar sobre qué hace el medio, incluso sobre dónde no los vemos.
En ese sentido, lo que se publica en la portada deja de ser relevante si tomamos en cuenta que mucha de la información consultada es indexada a través de los buscadores. Por tal motivo, la investigación trabajó con los códigos de sindicación de cada medio y con una tabla que tabulara y organizara diariamente la totalidad de los contenidos publicados.

Cabe mencionar también como un precursor de este tipo de estudios, la investigación emprendida por Xiaopeng Wang y Daniel Riffe, relacionada a una exploración de los tamaños de muestra para el análisis de contenido del sitio web del New York Times (2006). Para ese caso, la investigación se basó en demostrar la eficacia, o no, de un método para la observación y el análisis de contenidos en la prensa digital. La muestra abarcó un año en total y midió las famosas variables propuestas por G.H. Stempel (1989) para el análisis de contenidos que clasifica al periodismo en actos políticos y de gobierno, la guerra y la defensa, la diplomacia y las relaciones exteriores, la actividad económica, la agricultura, entre otras. Los resultados son interesantes dado el tiempo de medición. Por ejemplo, una de las conclusiones señala que el estudio demostró que el tamaño de la muestra, en este caso de seis días por semana, fue eficaz y que hay variables más 'sensibles' a otras, como por ejemplo lo "multimedia”.

3 El trabajo consistió en trasladar toda la información publicada diariamente a nuestro sistema para luego tabularla a partir de los indicadores de investigación. 
Un estudio mucho más reciente que podríamos incluir en esta revisión es el publicado el 2013 por Pablo Boczkowski y Eugenia Mitchelstein titulado "The News Gap: When the Information Preferences ofthe Media and the Public Diverge". Se trata de un estudio exploratorio de contenidos de sitios web de sietes países, The Guardian, CNN, El País, La Nación, entre otros. Los resultados a los que llegan son más que sugerentes. Por ejemplo, una de sus conclusiones señala que los usuarios de la prensa digital siguen prefiriendo las historias tradicionales cortas que respetan la clásica pirámide invertida a las historias más largas.

Lo más significativo de la publicación tiene que ver con el cuestionamiento sobre los retos de estos diarios para captar más audiencia. Según los autores, estos no deben optar por la solución de brindar contenidos más livianos de deportes y espectáculos, en detrimento de su prestigio periodístico. Justamente, en nuestra investigación nos propusimos encontrar si dicho prestigio ha dado paso a la búsqueda por la explosión de las visitas, de manera muy similar a como funciona el rating en la televisión. En ese sentido, el prestigio, rigor, verificación de datos y trabajo periodístico ha sido reemplazado por un número que dicta cuántas visitas se obtuvieron cada año, mes, día, hora e incluso minuto.

Cabe señalar como antecedentes también la bibliografía previa desarrollada por autores pioneros en analítica y arquitectura web, como lo son los trabajos de Jakob Nielsen y su famoso tratado de "Usabilidad de páginas de inicio: análisis de 50 Sitios Web" (2000), o de autores tales como Luis Codina, o estudios más recientes como el de Ben Shneiderman titulado "Designing the User Interface: Strategies for Effective Human-Computer Interaction" sobre interfaces web.

Finalmente, no hay que olvidar tampoco estudios vinculados a indagaciones de contenidos más de carácter cualitativo relacionados a social media de autores como Clay Shirky (2008) o Danah Boyd (2014). Estos últimos, sobre todo, a partir de publicaciones, en los que se destaca y cuestiona el real poder de la audiencia en la agenda de los medios de comunicación, así como los cambios de comportamientos generados en los jóvenes.

\section{Objeto de estudio: exploraciones preliminares}

Los primeros pasos del periodismo digital peruano datan del año 1995, cuando la revista semanal Caretas publicó por primera vez su página web. Luego le siguieron $L a$ República (septiembre, 1995), La Encuesta (noviembre, 1996), RPP (1996), El Tiempo y El Comercio (ambos en 1997). Estos datos son recogidos por Ciberperiodismo en el Perú (2008) de Lyudmyla Yezers'ka, una de las primeras publicaciones sobre la génesis del periodismo digital peruano. Esa publicación, además de presentar datos de carácter historicista basados en 
entrevistas con especialistas, es el primer acercamiento al periodismo digital peruano. Tomando como método el propuesto por Luis Codina, la autora basa su investigación de los principales diarios digitales peruanos (El Comercio, La República, $R P P$, entre otros) priorizando los aspectos de representación de acceso informativo, así como aspectos ergonómicos y de adecuación al medio digital (Salmón, 2009).

En la misma línea de la investigación de Yezers'ka podemos mencionar el trabajo de Liliana Sánchez, "Evolución del periodismo digital peruano. Análisis de los casos: Radio Programas del Perú, Panamericana.com y Terra.com (1995-2005)", en donde la autora indaga sobre los contenidos de tres de los primeros sitios de noticias peruanos. Sánchez aborda los objetos de estudio a partir de la observación básica y de datos muestrales basados en una plantilla de observación. Nuevamente, la información es interesante en la medida en que funciona como una primera imagen del periodismo digital peruano en su primera década de existencia.

Exploraciones más cercanas en relación a nuestro estudio son los de Gisella Salmón (2009), Sofía Pichihua (2012) y Alfredo Espinoza (2012). En el primer caso, Salmón desarrolla su tesis sobre la configuración del lenguaje ciberperiodístico, el desarrollo y las tendencias de la prensa digital peruana. Para ello, analiza los sitios El Comercio, RPP y la Agencia de Noticias Andina. Vinculada metodológicamente con el trabajo de Yezers'ka, en la utilización del método Codina de medición de adecuación al medio y también en las entrevistas con especialistas, el trabajo de Salmón constituye un espacio intermedio entre la preocupación por la arquitectura informativa y por los propios contenidos web. A su conclusión general, netamente formal, le siguen una serie de conclusiones específicas en donde los contenidos ya empiezan a aparecer.

Así, por ejemplo, dice: "Aunque se toma como insumo principal la información proveniente de radio, agencias y televisión, ya es posible notar un alto índice de notas propias, elaboradas desde la redacción digital” (Salmón, 2009). Esto puede entenderse si consideramos que la competencia por la generación de primicias para atraer la atención del lector se da también en Internet; por ello las primicias difundidas rápidamente ayudan al medio a posicionarse frente a sus pares.

Todas las investigaciones mencionadas se basan en un contexto en el que la imagen del periodista como intermediario entre la información y la audiencia es cuestionada. Tal como lo señala Baricco (2006), las nuevas tecnologías permiten que los usuarios puedan prescindir del intermediario (periodista, curador, programador radial o televisivo), teniendo la posibilidad de enfrentarse por sí solos a la información. Eso es Google, Facebook, Twitter o YouTube, espacios donde el propio usuario decide y define su propio 
trazo, y donde además hemos aprendido a organizarnos, por temas en común o cercanía (amigos y familiares), para compartir información y comunicarnos. Es en ese espacio donde el periodismo debe reacomodarse y comprender sus características: plataformas múltiples, medios híbridos, intertextuales, multimediales o transmediales (Scolari, 2008).

\section{Audiencias y medios estudiados}

Según las cifras del Interactive Advertising Bureau (IAB) Perú ${ }^{4}$ del 2014, año previo en el que se desarrolló la investigación, las web de noticias con más visitas del Perú eran, en este orden, El Comercio, La República y RPP.

Según estas cifras, para el mes de septiembre, El Comercio alcanzaba casi 13 millones de visitas únicas al mes, mientras $\mathrm{La}$ República y RPP llegaban aproximadamente a los 12 y 9 millones de visitas, respectivamente. Las cifras confirmaban que en poco tiempo los sitios incrementaron sus visitas, lo que marcaba una tendencia clara del crecimiento de la demanda de consumo de noticias por Internet, confirmada en la actualidad: en noviembre del 2018, El Comercio alcanzó los 44.1 millones de usuarios y 334 millones de páginas vistas, según registró su herramienta de medición interna Google Analytics 3605 .
Así pues, pasados casi 20 años de la aparición en el Perú de la prensa digital, el presente estudio buscaba indagar en el contenido. Estudiar el contenido de la prensa digital peruana es estudiar la raíz del supuesto problema de los que han reclamado el fin del periodismo. Sobre todo porque a la desintermediación propuesta por Castells (2001) o Piscitelli (2001), en el que los periodistas son cada vez menos necesarios, la respuesta de la industria en diversas partes del mundo ha sido dispar. The New York Time o The Washington Post, por ejemplo, optaron en algún momento por cobrar por sus contenidos, a la par de potenciar la generación de contenido de calidad propio, es decir, respetando los mismos preceptos del periodismo de siempre: de rigor, de confianza y de respeto al lector.

Hubo otros medios, en el mundo y también en el Perú, que optaron por la solución más "sencilla": publicar contenidos previamente hechos, sobre todo relacionados con los temas de espectáculos y deportes. De esa forma se apostó por la explosión de visitas. Los redactores entonces ya no buscaban temas que pudieran interesar sino temas que ya eran interesantes previamente. Así, por ejemplo, los videos más vistos de la semana en YouTube se convirtieron en parte del contenido de la prensa digital peruana o

4 El Interactive Advertising Bureau (IAB), sección Perú, desarrolló estudios de audiencia en el Perú hasta el año 2015, aproximadamente. Para más información, ver: "¿Cuáles son los medios digitales más leídos en el Perú?". Rescatado de https://bit.ly/2saQgFU

5 Ver: "El Comercio bate récords y ratifica su liderazgo como el medio peruano más visto en internet". Rescatado de https://bit.ly/2J3j2DQ 
los memes compartidos en redes sociales en notas periodísticas de gran demanda.

Este fenómeno se dio además en un contexto marcado por la discusión por la concentración de medios en el Perú, en el que el Grupo El Comercio maneja casi el 80\% del mercado de la prensa escrita peruana. Si partimos de la certeza de que no existe la variedad informativa a nivel de empresa, es aún más revelador constatar que en las versiones web, donde la audiencia ha crecido exponencialmente en los últimos años, como demuestran las cifras, también existe una peligrosa concentración de temas y estrategias que limitan al lector peruano. Creemos que verificar esto con un método y tener data es el primer paso para intentar comprender el fenómeno y plantear estrategias de cambio.

\section{Explicando la metodología}

El método aplicado consistió en un análisis de contenidos, para lo que se recogió toda la información publicada durante cuatro meses, de septiembre a diciembre del 2015, en las versiones digitales de los medios estudiados. En total, se registraron 69247 noticias, ubicándolas a partir de cuatro variables.

Las variables consideradas para el recojo de la información fueron las cuatro siguientes: sección, formato, género y fuente.

La variable "sección" estaba vinculada con la definición clásica de secciones periodísticas que divide la información según el tema tratado. De esta forma, se incluyeron las secciones propuestas por los mismos medios. Algunas de estas secciones fueron: política, internacional, deportes, economía, cultura, etc.

La variable "formato" indagaba en la materialidad de la noticia. Es decir, qué elemento era el que destacaba más en la información, sea este texto, imagen, audio, etc. Así, por ejemplo, en una galería de fotos, la imagen era lo destacado.

La variable "género" aludía a la reflexión sobre los géneros periodísticos más clásicos: informativo, interpretativo y de opinión. En ese sentido, el estudio, más allá de transferir información a nuestra base de datos, también supuso una revisión de cada nota para determinar a qué género pertenecía.

Finalmente, la variable "fuente" se vinculaba más con la procedencia de la información. Esta podía ser de elaboración propia, de agencia, de la televisión, redes sociales, etc.

Ejemplo de la ficha de registro:

\begin{tabular}{|c|c|c|c|c|c|c|}
\hline Día & Fecha & Medio & Sección & Género & Formato & Fuente \\
\hline & & & & & & \\
\hline
\end{tabular}


Figura 1. Producción total por cada medio

\begin{tabular}{|c|c|c|c|c|}
\hline & \multicolumn{5}{|c|}{ Producción por medio } \\
\hline & $\mathbf{2}$ & RPo & Total por mes \\
\hline Septiembe & 6117 & 6396 & 4553 & 17066 \\
\hline Octubre & 6114 & 6646 & 5416 & 18176 \\
\hline Noviembre & 5923 & 6765 & 4408 & 17096 \\
\hline Diciembre & 5960 & 6226 & 4723 & 16909 \\
\hline Total por medio & 24114 & 26033 & 19100 & 69247 \\
\hline
\end{tabular}

Elaboración propia

\section{Resultados}

Sobre la variable "sección": ¿qué es lo que más publican los medios digitales estudiados?

La sección con mayor cantidad de noticias fue la de temas nacionales ( $27.2 \%$ de los contenidos). En un segundo nivel se observa una producción pareja de contenidos asociados a las secciones internacional $(16.8 \%)$, deportes $(15.4 \%)$ y entretenimiento $(15.1 \%)$

Existe una producción regular de contenidos en los segmentos de política, Lima y economía, excluyendo el horóscopo. Las temáticas relacionadas a cultura $(1.2 \%)$, salud $(1.1 \%)$ y gastronomía (0.5\%) son las que cuentan con menor representación.

Figura 2. Total de noticias publicadas en las webs de El Comercio, La República y Radio Programas del Perú $(R P P)$, según la categoría "sección", durante los meses de septiembre a diciembre del 2015.

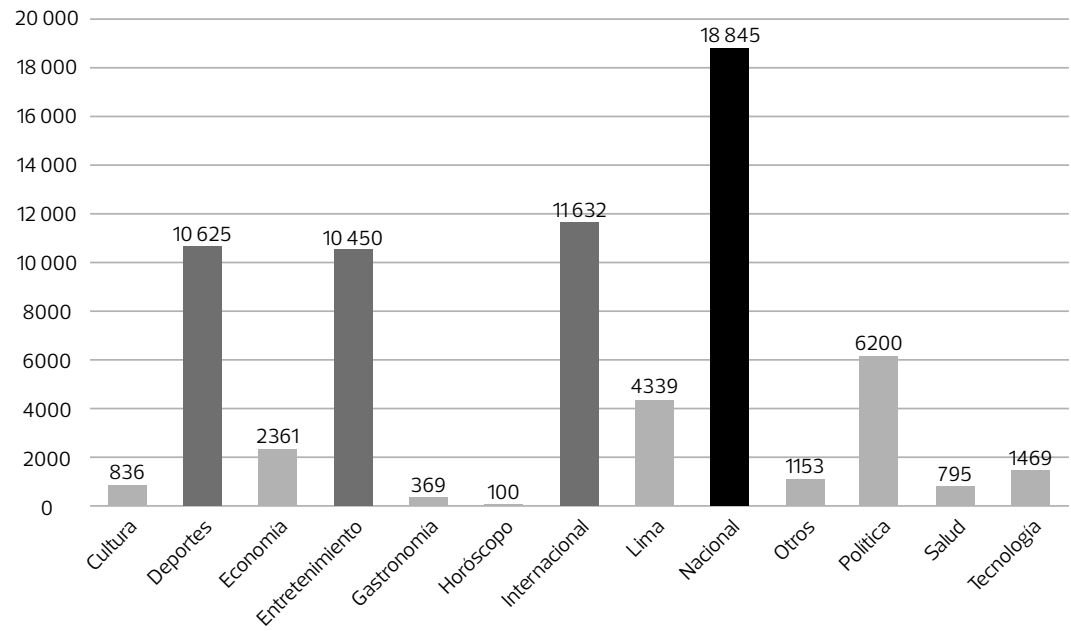

Elaboración propia. 


\section{El Comercio}

Para el caso de El Comercio, la sección con más publicaciones es la de temas internacionales $(24.1 \%)$. Luego existe paridad en la cantidad de noticias para las secciones de entretenimiento $(15.8 \%)$, deportes $(15.1 \%)$ y nacionales $(13.7 \%)$. En un tercer nivel se ubican las secciones de política (9.1\%), Lima $(7.5 \%)$ y, con una presencia ligeramente menor, las noticias sobre economía (5\%).

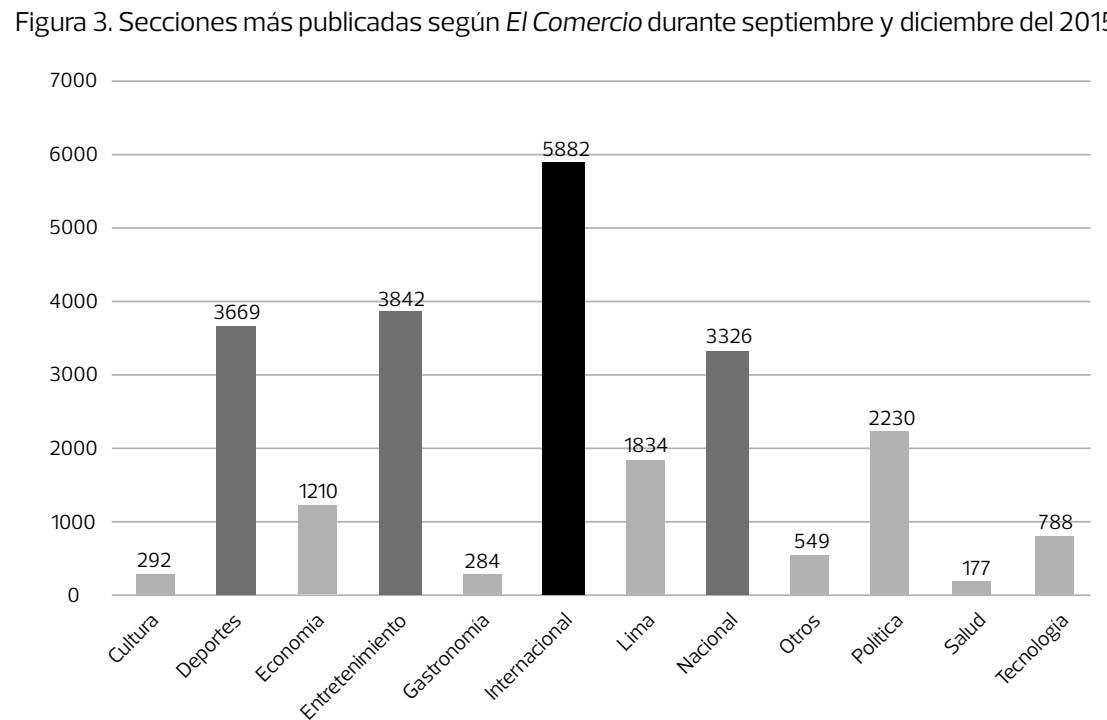

Elaboración propia.

\section{La República}

La versión web de La República prioriza fuertemente las noticias de índole nacional (34.1\%). Por debajo se ubican en un similar nivel de producción los contenidos sobre entretenimiento $(15.3 \%)$, deportes $(14.4 \%)$, temas internacionales $(12.3 \%)$ y política (10.7\%). Excluyendo los contenidos sobre Lima (5.6\%), el resto de las secciones cuentan con muy pocas actualizaciones. 
Figura 4. Secciones más publicadas según La República, durante septiembre y diciembre del 2015

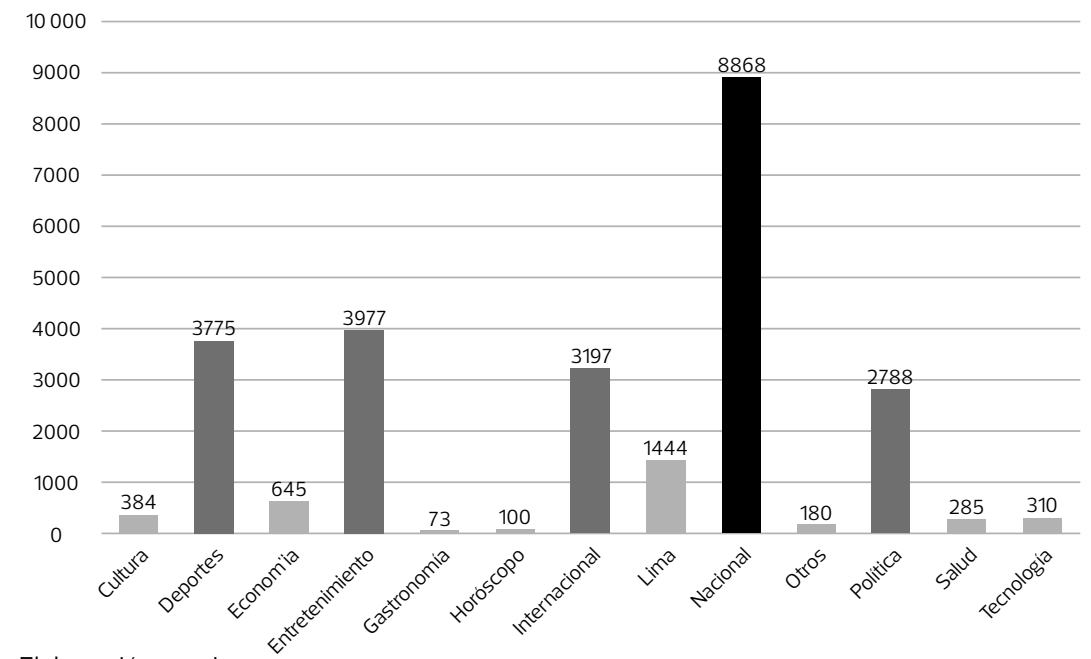

Elaboración propia.

\section{$\boldsymbol{R P P}$}

La web de esta radio peruana cuenta con una producción por secciones muy similar a la de La República. Predominan en ella los contenidos nacionales (34.9\%). Por debajo existe una ligera tendencia a priorizar los contenidos deportivos (16.8\%), respecto al entretenimiento (13.8\%) y temas internacionales (13.4\%). Las secciones de Lima (5.6\%) y política (6.2\%) cuentan con una regular cantidad de publicaciones.

Figura 5. Secciones más publicadas según RPP durante septiembre y diciembre del 2015

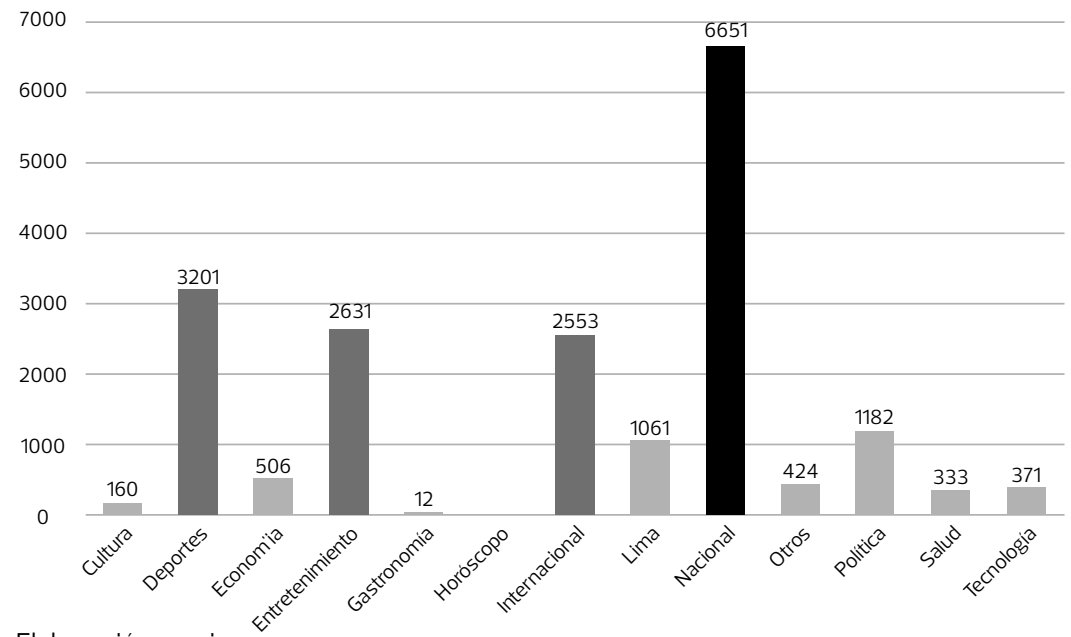

Elaboración propia. 
Para las fechas del estudio, la principal diferencia entre medios es la preferencia de El Comercio por noticias internacionales, a diferencia de La República y RPP, quienes privilegian temas nacionales. Estos dos últimos medios cuentan con una producción de contenido muy similar, solo con una ligera tendencia general en producir más contenido de parte de La República. Esta semejanza podría ser un primer indicio sobre la similitud de contenidos entre medios.

Figura 6. Comparativo de publicaciones por sección de los tres medios estudiados, entre septiembre y diciembre del 2015

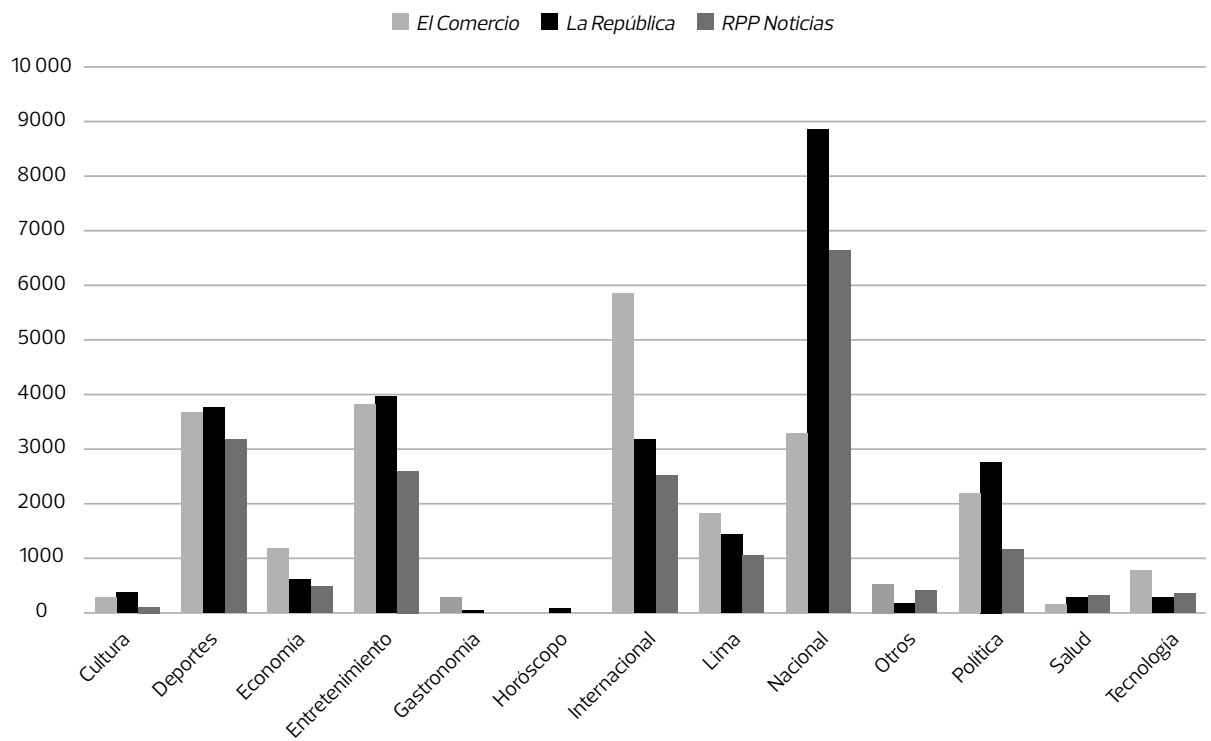

Elaboración propia.

$\underline{\text { Sobre la variable "fuente": ¿de dónde provienen las noticias publicadas? }}$

Para la fecha de estudio, los medios analizados suelen publicar mayoritariamente notas propias, producidas exclusivamente para sus plataformas digitales (29.5\%). Sin embargo, también suelen utilizar con frecuencia como fuente sus ediciones impresas $(20 \%)$.

En caso de no ser así, los canales de preferencia para obtener contenidos son otras webs $(18.3 \%)$ y las redes sociales $(14.4 \%)$. En menor medida, pueden considerar utilizar contenidos que provienen de un agencia de noticias $(7.3 \%)$ o de la televisión $(8.1 \%)$. 
Figura 7. Total de noticias publicadas en las webs de El Comercio, La República y RPP, según la categoría "fuente", durante los meses de septiembre a diciembre del 2015

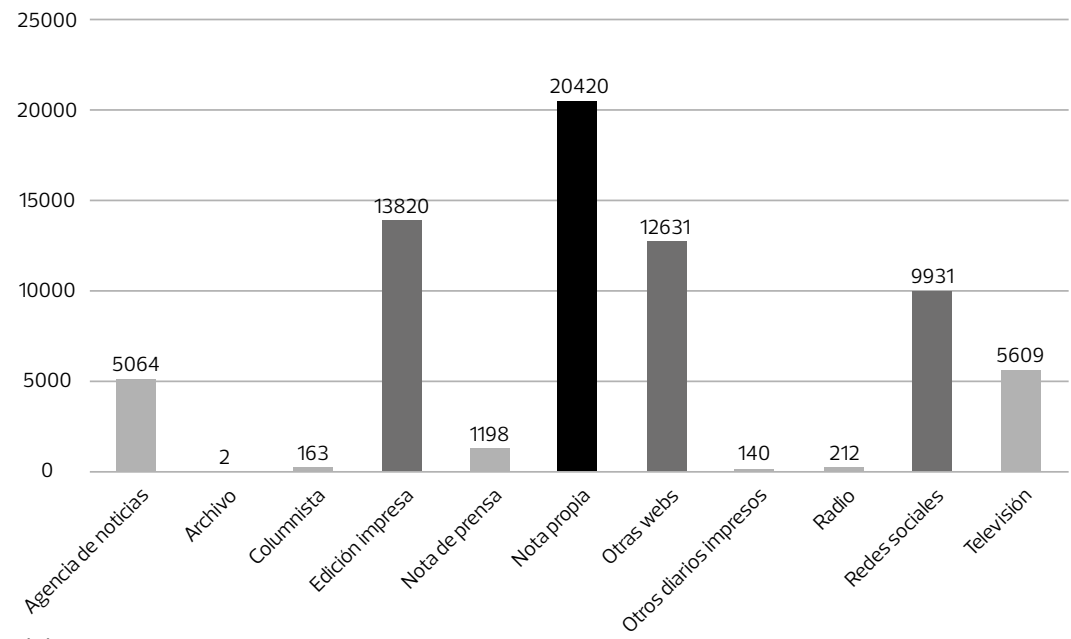

Elaboración propia.

\section{El Comercio}

Si bien este medio privilegia notas propios (31.2\%), su fuente principal está cerca de igualarse con las publicaciones que se basan en contenidos difundidos en otras webs (27.9\%). Su tercera fuente más utilizada son las redes sociales (19.6\%), seguidas mucho más atrás de las agencias de noticias $(7.8 \%)$ y de contenidos televisivos (10.5\%).

Figura 8. Fuentes más utilizadas según El Comercio durante septiembre y diciembre del 2015

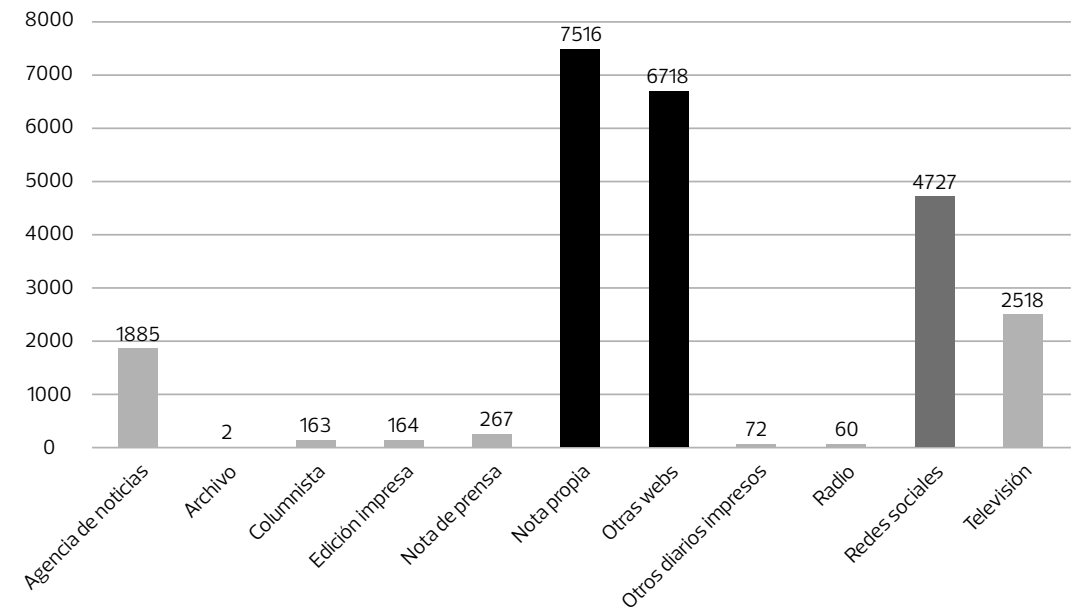

Elaboración propia. 


\section{La República}

En el caso de La República, la edición impresa (52.5\%) es la principal fuente de contenido para su plataforma digital. El medio hace uso también del contenido de otras webs $(12.4 \%)$, de redes sociales (11\%) y de notas propias (11.6\%) para complementar su oferta.

Figura 9. Fuentes más utilizadas según La República durante septiembre y diciembre del 2015

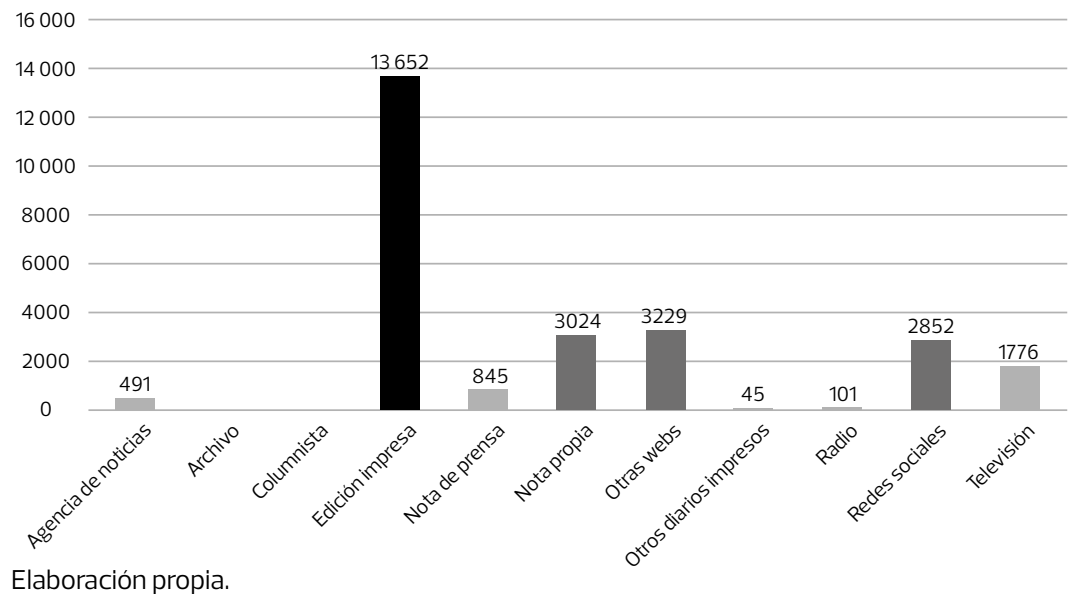

$\boldsymbol{R P P}$

Esta radio produce (nota propia) la gran mayoría del contenido que publica en su plataforma (51.8\%). También recurre, aunque en menor medida, a agencias de noticias $(14.1 \%)$, otras webs $(14.1 \%)$ y redes sociales (12.3\%).

Figura 10. Fuentes más utilizadas según RPP durante septiembre y diciembre del 2015

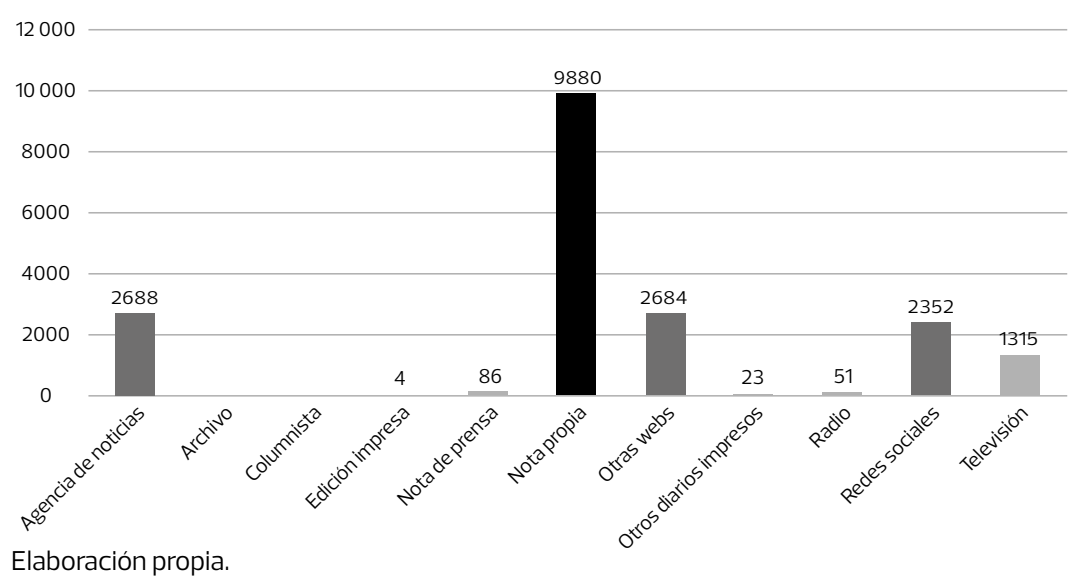

Elaboración propia. 
En base a lo observado, podemos decir que, durante las fechas de estudio, existen grandes diferencias entre los medios analizados respecto a las fuentes utilizadas para sus contenidos.

Si bien El Comercio utiliza mayormente notas propias, esta cantidad es superada por la suma de los contenidos externos utilizados, siendo este el medio que más usa como fuente otras webs y las redes sociales. Por su parte, La República es el medio que recoge la mayoría de su contenido de su edición impresa. Finalmente, $R P P$ es quien tiene mayor producción de notas propias, siendo estas las que constituyen la principal fuente de su contenido. Como apoyo, utiliza contenidos de otras webs, redes sociales, agencias de noticias y televisión. Ningún contenido proviene de su edición impresa ya que no cuenta con una.

Figura 11. Comparativo de publicaciones según fuente para los tres medios estudiados, entre septiembre y diciembre del 2015

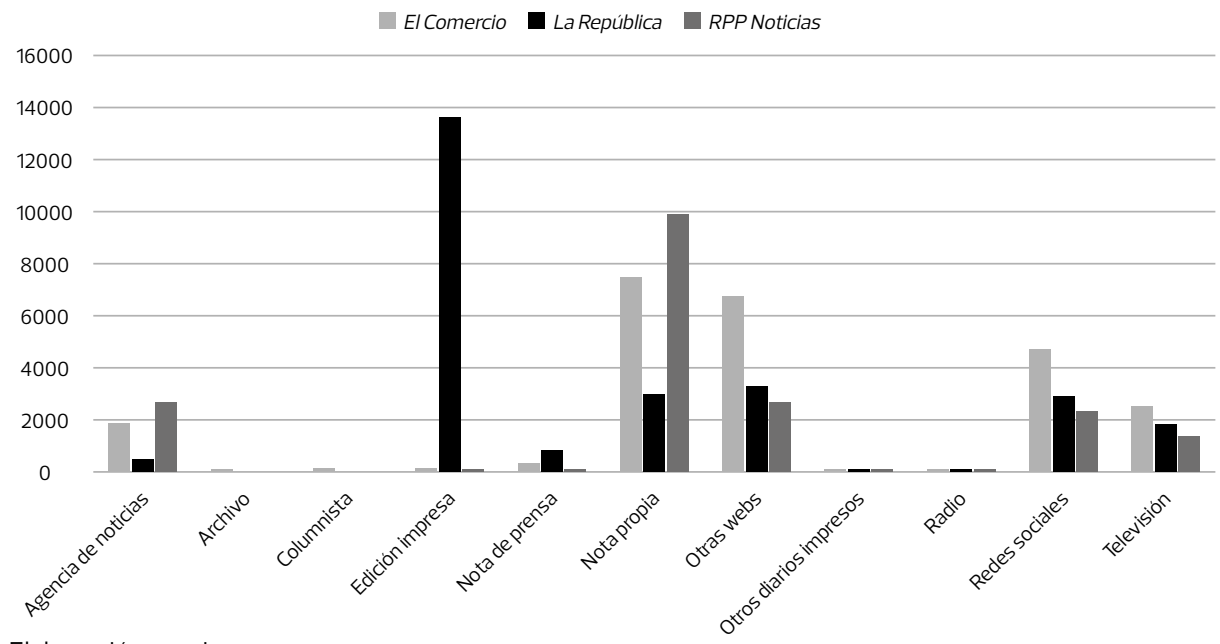

Elaboración propia.

\section{A manera de conclusiones}

Podemos afirmar que en la muestra existe una fuerte jerarquía a nivel de las secciones que los tres medios estudiados parecen seguir: 1. Nacional, 2. Deportes, 3. Entretenimiento y 4. Internacional. Lo político aún permanece relegado.
Las fuentes utilizadas varían mucho, acorde al momento y al medio, por lo que parece difícil establecer una conclusión definitiva. Sin embargo, se pueden observar ciertas tendencias:

- La República funcionó durante dichos meses principalmente como 
replicador de los contenidos difundidos en su versión impresa.

- Si bien las notas propias suelen ser, en general, la primera fuente utilizada por los medios estudiados para su sección digital, estas son solo uno de los elementos que contribuyen a su contenido diario.

- La mayoría de la información recogida de fuentes terceras proviene de redes sociales y de otras webs. Canales tradicionales para obtener información como las agencias de noticias y la televisión solo son considerados como fuentes secundarias.

- Las secciones de espectáculos y deportes no son de los temas más actualizados, a pesar de contar con un lugar importante en el contenido cotidiano de los medios estudiados. Son las temáticas con mayor cantidad de publicaciones detrás de las noticias nacionales en La República y $R P P$, y detrás de las noticias internacionales en el caso de El Comercio.

- Existe gran similitud en los contenidos de cada uno de los tres medios. Resulta fácil identificar una agenda común que orienta las publicaciones, con una manera de presentar similar en cada canal estudiado.

- Las estrategias a nivel de agenda de temáticas se muestran muy similares entre cada medio estudiado, lo que pasa en parte por explotar los mencionados segmentos de espectáculos y deportes.

- Si bien la tendencia general establece las notas propias como la primera fuente de información, estas solo conforma el $29.5 \%$ de la totalidad de los contenidos. El resto de las publicaciones proviene de otras webs, de las redes sociales y, en menor medida, de agencias de noticias y de la televisión.

- Ahora bien, a pesar de esta tendencia, existen fuertes diferencias según el medio referido. La plataforma que produce la menor cantidad de notas propias web es La República, situación que se explica por el fuerte uso de contenidos de la versión impresa. El Comercio se ubica en una posición media, considerando su propio contenido como una fuente más, entre otras. Finalmente, RPP parece priorizar esta producción interna para la gran mayoría de sus publicaciones.

Finalmente, creemos que los datos aquí presentados pueden servir como un punto de partida no solo para futuras investigaciones sobre el periodismo digital peruano, sino también pueden llevarnos a cuestionar el propio quehacer de la práctica periodística en nuestro país. Conocer las características del contenido nos permite también indagar en las estra- 
tegias de los medios a lo largo de estos últimos 20 años para subsistir al Internet. Por otro lado, las cifras dan cuenta de una necesidad renovadora que incorpore otros indicadores en la medición de resultados de los medios periodísticos, por ejemplo de carácter más cualitativo, que nos permitan centrarnos en lo inalterable del periodismo: el contenido de calidad y la verdad.

\section{REFERENCIAS}

Acevedo, J. (2013). La concentración mediática no es un asunto de privados. Quehacer, (191), 64-69.

Baricco, A. (2006). Los bárbaros. Ensayo sobre la mutación. Barcelona: Anagrama.

Boczkowski, P. y Mitchelstein E. (2013). The News Gap: When the Information Preferences of the Media and the Public Diverge. Cambridge: MIT Press. https://doi.org/10.7551/mitpress/9780262019835.001.0001

Boyd, D. (2014). It's Complicated: The Social Lives of Networked Teens. New Haven / Londres: Yale University.

Castells, M. (2001). La Galaxia Internet. Reflexiones sobre Internet, empresa y sociedad. Barcelona: Areté. https:// doi.org/10.1007/978-3-322-89613-1

Codina, L. (2000). Metodología de análisis y evaluación de recursos digitales en línea. Barcelona: Col-legi Oficial de Bibliotecaris - Documentalistes de Catalunya (Cobdc). Recuperado de http://tiny.cc/ml4e8y

Kerlinger, F. (1979). Investigación del comportamiento. México D.F.: Interamericana.
Nielsen, J. (2000). Usabilidad de páginas de inicio: análisis de 50 Sitios. Madrid: Web Pearson Educación.

Odriozola, J.; López, G.; Llorca, G. y De la Fuente, M. (Mayo del 2010). Metodologías de análisis de contenido en los cibermedios españoles: el caso del diario abc.es. Comunicación presentada en el XII Congreso de la Sociedad Española de Periodística, Universidad CEU Cardenal Herrera, Valencia.

Odriozola, J.; López, G.; Llorca, G. y De la Fuente, M. (Julio del 2011). Content Analysis applied to Digital Media: a Comparison of News in the Guardian, Clarín, and Asahi Simbun. Journalism Studies Section Conference. Comunicación presentada en ECREA 2011, Universidad de Navarra, Pamplona.

Pichihua, S. (2012). Producción de noticias en las redacciones digitales de Perú.21 y Depor (tesis de licenciatura en Comunicaciones con mención en periodismo). Pontificia Universidad Católica del Perú (PUCP), Lima.

Piscitelli, A. (2001). La Generación Nasdaq: Apogeo (y ¿derrumbe?) de la Economía Digital. Buenos Aires: Granica.

Ruiz, O. J. I. (2012). Metodología de la investigación cualitativa. Bilbao: Universidad de Deusto.

Salmón, G. (2009). Del papel a la red: la configuración del lenguaje ciberperiodístico, desarrollo y tendencias de la prensa digital peruana: análisis de casos: El Comercio, RPP y la Agencia de Noticias Andina (tesis de licenciatura en Comunicaciones con mención en periodismo). Pontificia Universidad Católica del Perú (PUCP), Lima.

Sánchez, L. (2007). Evolución del periodismo digital peruano. Análisis de los casos: rrp.com, el comercioperu.com, 
panamericana.com y terra.com (1995-2005) (tesis de licenciatura en Comunicaciones con mención en Periodismo). Universidad de Ciencias Aplicadas, Lima. Recuperado de https://bit.ly/2M2otSW

Scolari, C. (2008). Hipermediaciones. Elementos para una Teoría de la Comunicación Digital Interactiva. Barcelona: Gedisa Editorial.

Shirky, C. (2008). Here Comes Everybody: The Power of Organizing Without Organizations. New York: Penguin Press.

Stempel, G. H. (1989). Content analysis. In G.H. Stempel and B.H. Westley (Eds) Research methods in mass communications. New Jersey: Prentice-Hall.

Wang, X. y Riffe, D. (2006). An Exploration of Samples Sizes for Content Analysis of the New York Times Web Site. Web Journal of Mass Communication Research, 20.

Yezers'ka, L. (2008). Ciberperiodismo en el Perú. Análisis de los diarios digitales. Lima: Editorial San Marcos. https://doi.org/10.4185/RLCS-63-2008754-071-082 